\title{
Expectancy and frequency effects on perceptual and motor systems in choice reaction time
}

\author{
JEFF MILLER \\ University of California at San Diego, La Jolla, California 92093
}

and

\author{
RAN ANBAR \\ Pritzker School of Medicine, University of Chicago, Chicago, Illinois 60637
}

\begin{abstract}
Three experiments investigated the effects of expectancy and stimulus frequency in character classification and identification tasks. Both expectancy and frequency were found to have effects on overall response latency, but the two factors had different patterns of interaction with two other experimental variables. The effect of visual quality was larger for low-frequency stimuli than for high-frequency stimuli, whereas the effect of quality was independent of expectancy. The effect of S-R compatibility was more strongly influenced by whether a stimulus was expected than by the frequency of the stimulus. The results suggest that expectancy and frequency have somewhat different effects on human information processing mechanisms. Expectancy allows temporary preparation for a specific S-R pair in response selection processes, but it has no effect on perceptual processes. Stimulus frequency, on the other hand, influences the efficiency of both perceptual and response selection processes. The overall effect of stimulus probability may be interpreted as having as components both expectancy and frequency effects.
\end{abstract}

It has often been observed that people make faster and more accurate discriminative responses to stimuli that occur with relatively high probability than to stimuli that occur with relatively low probability (e.g., Fitts, Peterson, \& Wolpe, 1963; Hyman, 1953; LaBerge \& Tweedy, 1964). Phenomena of this kind, generally referred to as "stimulus probability effects," have been investigated extensively because of their importance for theories of reaction time (RT) in speeded choice tasks.

This article addresses two questions emerging from the study of stimulus probability effects and attempts to show that they are related. The first question concerns the extent to which probability effects can be explained by the tendency to expect stimuli that are more probable: Does frequency of stimulus occurrence itself produce any effect, or does it only produce an indirect effect through the control of subjective expectancies? The second question concerns the locus of probability effects: What mental processes are influenced when probability is varied? Previous studies have examined these questions separately, and the present studies were designed to extend the results of the previous studies by looking at the two questions together.

When a stimulus is presented with high probability,

This research was supported by NSF Grant BNS-7824772 to the first author. Requests for reprints should be addressed to Jeff Miller at the Department of Psychology, C-009, University of California-San Diego, La Jolla, California 92093 observers come to expect the stimulus on a high proportion of trials (Estes, 1964). Expectancy for an item is known to produce a sizable processing advantage for that item (e.g., Beller, 1971; Bernstein \& Reese, 1965; Klatzky \& Smith, 1972; Neely, 1977), and some theorists (e.g., Laming, 1969; Theois, Smith, Haviland, Traupmann, \& May, 1973) have suggested that expectancy might be entirely responsible for stimulus probability effects.

Although expectancy is surely one of the factors responsible for probability effects, some evidence indicates that frequency of occurrence also plays a role. Geller and his colleagues (Geller \& Pitz, 1970; Geller, Whitman, \& Post, 1973; Geller, Whitman, Wrenn, \& Shipley, 1971) observed expectancies by asking subjects to predict which stimulus would be presented on each trial. Responses were much faster to expected than to unexpected stimuli, but RT also varied slightly as a function of stimulus frequency, even for correctly predicted stimuli. Similar results were reported by Hinrichs and Craft (1971). These results are not surprising, given the view that processing of a stimulus is more efficient the more frequently it is tested, due to practice and learning effects.

The results of many attempts to determine which mental processes are influenced by stimulus probability indicate that probability has several different loci of effect. One set of studies used many-to-one S-R mappings in order to unconfound stimulus probability 
and response probability. To the extent that probability influences stimulus recognition mechanisms, it was argued, there should be an effect of stimulus probability with response probability held constant. To the extent that probability affects response processes, there should be an effect of response probability with stimulus probability held constant. Significant results were obtained both for stimulus probability (Bertelson \& Tisseyre, 1966; Hawkins, Thomas, \& Drury, 1970; Orenstein, 1970 ) and for response probability (Dillon, 1966; Keele, 1969), and several studies obtained both effects within a single experiment (Bernstein, Schurman, \& Forester, 1967; Biederman \& Zachary, 1970; Hawkins, MacKay, Holley, Friedin, \& Cohen, 1973; LaBerge, Legrand, \& Hobbie, 1969).

Another approach to the question of which processes are influenced by probability is to examine interactions of probability with other experimental variables. Under a variety of circumstances, for example, the effect of visually degrading a stimulus is larger for low-probability stimuli than for high-probability stimuli (e.g., Miller \& Pachella, 1973, 1976). This finding suggests that probability influences the time required by perceptual processes to recognize a stimulus. Similarly, the effect of S-R compatibility is generally larger for low-probability stimuli than for high-probability stimuli (e.g., Hawkins et al., 1973; Sanders, 1970; Spector \& Lyons, 1976), suggesting that probability also affects the time needed for response selection. Indeed, Blackman (1975) obtained interactions of probability with both stimulus quality and S-R compatibility in a single experiment, although these variables were additive with each other.

Given that expectancy and frequency both contribute to the effect of probability and given that probability affects both recognition and response processes, it seemed logical to look separately at effects of expectancy and frequency on recognition and response processes. The experiments reported here were designed to separate the effects of expectancy and stimulus frequency and to examine separately the interactions of these variables with S-R compatibility and stimulus quality. The goal was to discover differences in the influence of expectancy and frequency on stimulus recognition and response selection processes.

\section{EXPERIMENT 1}

This experiment examined the relationship among the variables of expectancy, stimulus frequency, and S-R compatibility. As noted in the introduction, it is well established that S-R compatibility has a larger $\mathrm{etfect}$ for low-probability stimuli than for high-probability stimuli. This experiment was designed to find out whether expectancy and frequency each modify the compatibility effect or whether the Probability by Compatibility interaction can be attributed to one of these factors alone.
Some evidence suggests that both expectancy and frequency should interact with S-R compatibility. Fitts (1964) found that compatibility effects decrease after practice, suggesting that stimulus frequency should interact with compatibility. Rosenbaum (1980) has shown that response selection and initiation processes benefit greatly from an opportunity for advance preparation, suggesting that expectancy should also interact with compatibility.

Expectancies were controlled in this experiment (as well as Experiments 2 and 3) through the use of hints. On each trial, the subject was given a hint as to the letter that would be tested. Stimulus frequency was varied orthogonally with the hint manipulation. By covarying these two factors in a single experiment, the separate effects and interactions of the two factors can be studied. Several considerations suggest that subjects would base their expectancies on the hints rather than on stimulus frequency. LaBerge, Van Gelder, and Yellott (1970) have shown that the use of hints eliminates the sequential effects normally found in RT experiments, and they concluded that the hints were effective at controlling expectancies. Furthermore, it is reasonable that the hints would control expectancies, since the hints correctly predicted the test letters with much greater reliability than stimulus probability did. Finally, the hints provided specific prompting for a given item, naturally drawing the subjects' attention to that item (cf. Neely, 1977).

\section{Method}

Subjects. Twenty-four right-handed undergraduate students at the University of California, San Diego, served as subjects in this experiment. Each subject served in a single experimental session of about $30-40 \mathrm{~min}$ and partially fulfilled an introductory psychology course requirement by participating in the experiment.

Apparatus and Stimuli. Stimuli were presented and responses and response latencies were recorded by an Automated Data Systems Micro-8 computer. Stimuli were the letters A through $\mathbf{F}$, displayed on an oscilloscope screen located about $50 \mathrm{~cm}$ from the subject. Subjects were seated in a dimly lit room, and stimuli were light figures on a dark background. Stimulus letters were drawn with solid lines and were about $3 \mathrm{~cm}$ high. Responses were made on a microswitch keyboard located directly in front of the subject, with the index, middle, and ring fingers of both hands.

Procedure. Each subject was tested in four blocks of 104 trials. For two of the blocks, the stimulus letters were assigned to response keys in the natural order of $A, B, C, D, E$, and $F$, from left to right (compatible condition). For the other two blocks, the stimulus letters were assigned to response keys, from left to right, in the order E, F, D, C, A, and B (incompatible condition). Half of the subjects first received two blocks in the compatible condition, followed by two blocks in the incompatible condition; the other half of the subjects were tested in the incompatible condition first.

On each trial, the subject was first presented a hint as to the identity of the test letter that would follow. These hints were correct $75 \%$ of the time, and subjects were informed of this fact. Trials on which the hint was actually correct will be referred to as trials in the "expected" condition, and trials on which the hint was incorrect will be referred to as trials in the "unexpected" condition. In order to maximize expectancy effects, 
subjects were explicitly asked to try to prepare for the stimulus that the hint indicated. The hint remained on the screen for $.5 \mathrm{sec}$, and there was a $1-\mathrm{sec}$ delay between the offset of the hint letter and the onset of the test letter. The test letter remained on the screen until the subject made a response, at which time it disappeared and accuracy feedback was given. About $1.5 \mathrm{sec}$ later, the next hint was presented.

The six stimulus letters were both presented as hints and tested unequally often. The frequencies of test were $31 \%, 15 \%$, and $4 \%$, and two stimulus letters were assigned to each frequency. In order that every hint letter would have a $75 \%$ chance of being correct, frequency of use as a hint letter had to be equal to test frequency. Thus, if the letter " $A$ " was tested on $15 \%$ of the trials, it was also used as a hint on $15 \%$ of the trials.

The test frequency associated with each stimulus letter remained constant across the four blocks for a given subject even when compatibility was switched. In addition, test frequencies were assigned to letters so that the frequency of a particular response did not change between the compatible and incompatible conditions for a given subject. This was accomplished by assigning equal probabilities to the letters in the following pairs: $(A, E),(B, F),(C, D)$. Since the mapping for the incompatible condition was obtained from the compatible mapping by interchanging the letters in these pairs, the probability of a particular response did not change between the compatible and incompatible conditions. The purpose of this constraint was to reduce the error variability in the Compatibility by Stimulus Frequency interaction by removing differences due to differential speeds of the different fingers. Within these constraints, the assignment of letters to probabilities was counterbalanced across subjects.

\section{Results and Discussion}

For each block of trials, the average RT and percentage of correct responses (PC) were computed for each test letter both in the expected and the unexpected conditions. Figure 1 shows the average RT as a function of expectancy, frequency, and S-R compatibility. An analysis of variance confirmed the reliability of the main effects and interactions apparent in Figure 1. The main effects of compatibility $[\mathrm{F}(1,22)=51, \mathrm{p}<.01, \mathrm{MSe}=$ $50,180]$, frequency $[\mathrm{F}(2,44)=21, \mathrm{p}<.01, \mathrm{MSe}=$ $36,299]$, and expectancy $[F(1,22)=330, p<.01$, $\mathrm{MSe}=116,850]$ were all highly significant. Furthermore, the effect of compatibility was larger for unexpected than for expected stimuli $[\mathrm{F}(1,22)=33, \mathrm{p}<.01$, $\mathrm{MSe}=39,347]$, and the effect of compatibility was larger for low-frequency stimuli than for high-frequency stimuli $[F(2,44)=3.89, p<.05, \quad \mathrm{MSe}=27,969]$. In addition, the effect of frequency was larger for unexpected stimuli than for expected stimuli $[F(2,44)=$ $51, \mathrm{p}<.01, \mathrm{MSe}=19,081]$. Finally, the three-way interaction of these factors was significant $[F(2,44)=$ $3.96, \mathrm{p}<.05, \mathrm{MSe}=26,705]$. A similar analysis of PC did not suggest that any of the results might have been due to speed-accuracy tradeoffs. The largest difference was between expected (96.6) and unexpected (90.5) trials $[F(1,22)=22, p<.01, \mathrm{MSe}=476]$. The effect of stimulus frequency was also significant $[F(2,44)=4.86$, $p<.01, \mathrm{MSe}=158]$, with fewer errors being made to the more frequent test letters. Neither the main effect of compatibility nor any of the above interactions approached significance.

Figures 2 and 3 show the separate interactions of compatibility with expectancy and of compatibility with stimulus frequency. As is apparent from the figures, the interaction of compatibility with expectancy was somewhat larger than the interaction of compatibility with frequency. The compatibility effect was only $27 \mathrm{msec}$ for expected stimuli and increased to $163 \mathrm{msec}$ for unexpected stimuli. The compatibility effect was $61 \mathrm{msec}$ for high-frequency stimuli and $128 \mathrm{msec}$ for

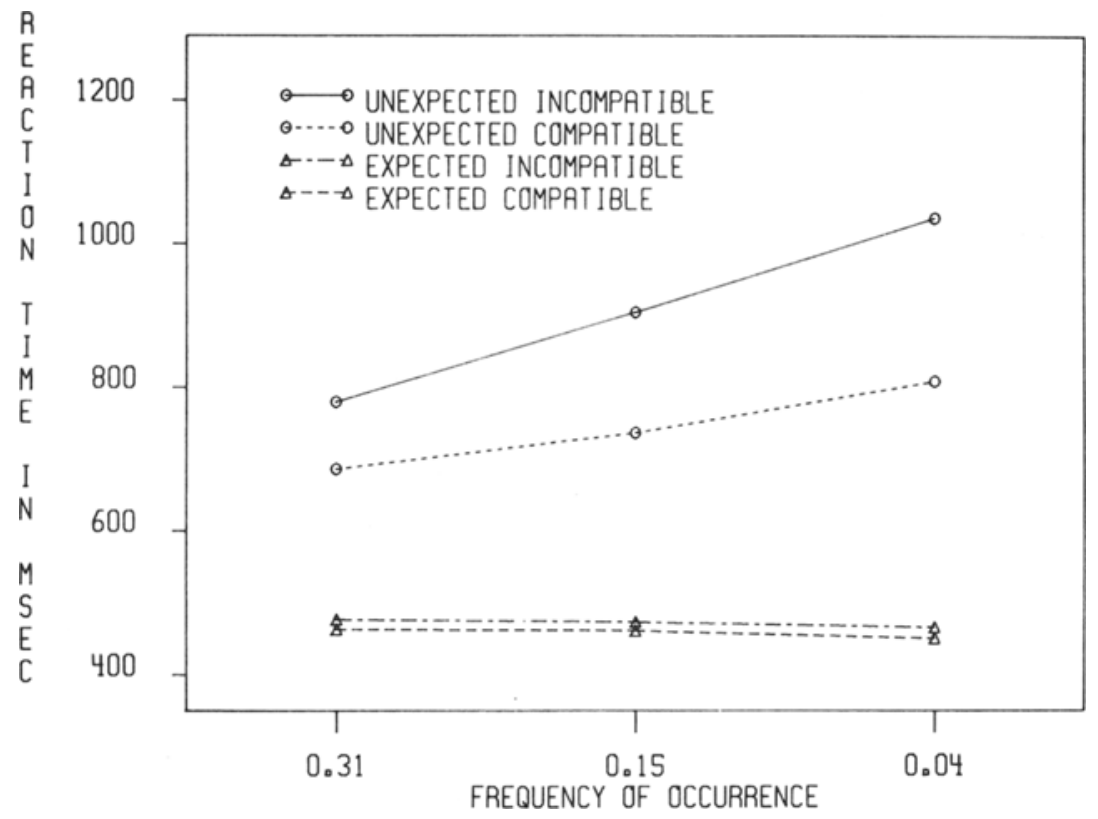

Figure 1. Experiment 1. Reaction time as a function of expectancy, stimulus frequency, and S-R compatibility. 


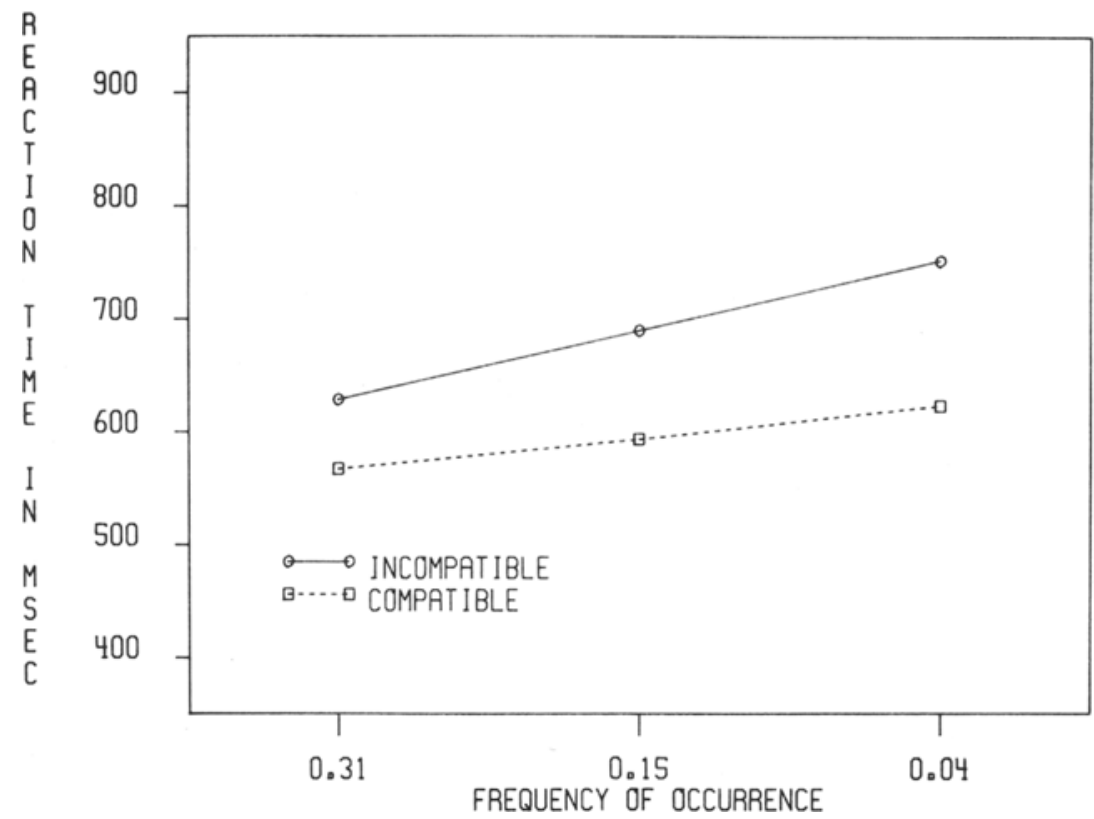

Figure 2. Experiment 1. Reaction time as a function of frequency of occurrence and S-R compatibility.

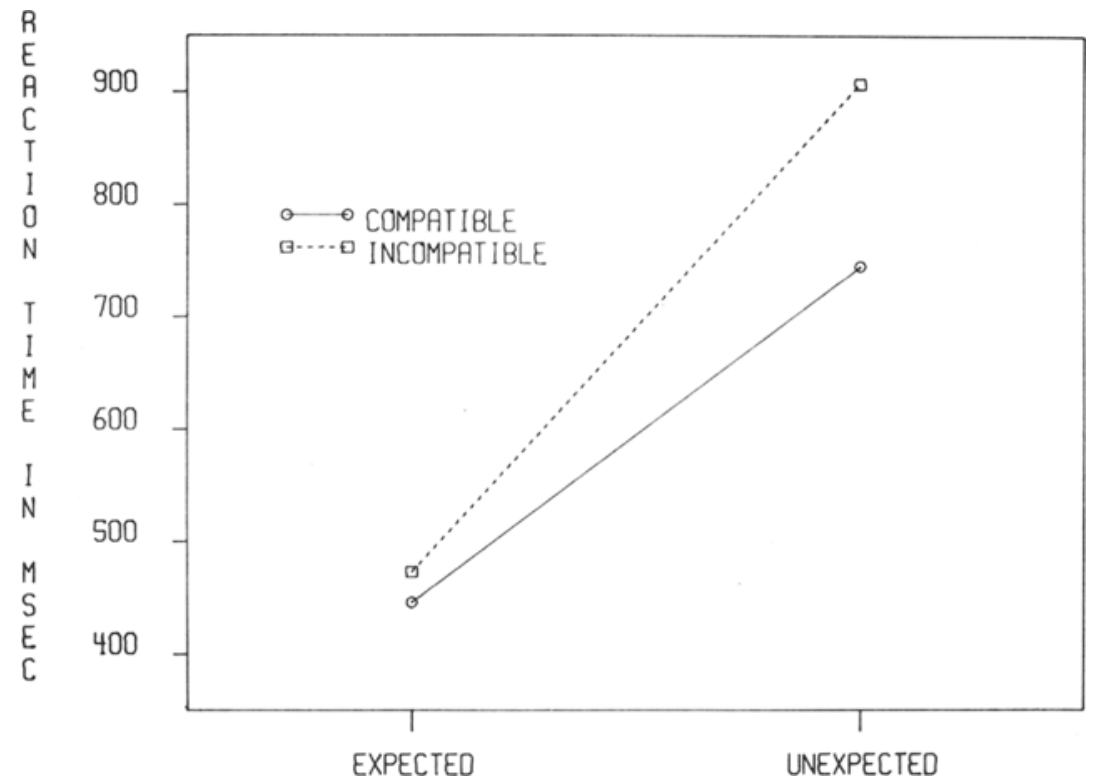

Figure 3. Experiment 1. Reaction time as a function of expectancy and S-R compatibility.

low-frequency stimuli. The difference in the compatibility effect between expected and unexpected stimuli was reliably larger than that between high- and lowfrequency stimuli $[t(23)=2.83, p<.05]$. These results suggest that the effect of S-R compatibility depends more on whether a stimulus is expected than on the relative frequency of the stimulus. This conclusion can only be tentative, since it depends on both the particular expectancy manipulation and the range of stimulus relative frequencies. Since these were typical of research in the area, though, the conclusion probably applies to most previous studies.

It is possible to try to reconstruct from these data the probability effect usually observed in tasks in which subjects are not given hints. The stimuli that were both high frequency and expected should correspond to "high- 
probability" stimuli, whereas unexpected low-frequency stimuli should correspond to "low-probability" stimuli. Table 1 shows mean RTs and PCs as a function of this pseudoprobability manipulation and of S-R compatibility. The main effect for probability is much larger here than is generally observed, possibly because highprobability stimuli are not normally $100 \%$ expected. The usual interaction of probability and compatibility is replicated, however, supporting the idea that probability can be decomposed into expectancy and frequency components.

The finding of no frequency effect for expected stimuli (see Figure 1) is surprising in terms of the ubiquity of results showing an effect of frequency even for expected stimuli (Geller \& Pitz, 1970; Geller et al., 1971, 1973; Hinrichs \& Craft, 1971; but see Hinrichs, 1970). Of course, it is possible that there really is such an effect, but that the present experiment lacked power sufficient to demonstrate it. It is also possible that having stimuli appear unequally frequently as hints obscured the effect, if subjects prepared more extensively after being given an infrequent hint. The previously reported effect could also be artifactual, however. In the earlier studies, subjects were asked to predict which stimulus would appear on each trial. It is possible that subjects predict low-frequency stimuli with less confidence than they predict high-frequency stimuli, and preparedness could vary as a function of confidence in the prediction.

If frequency really does not affect RT to expected stimuli, it is possible to explain the effects observed in Experiment 1 using only the concept of expectancy. It must be assumed, first, that responses to expected stimuli are faster than responses to unexpected stimuli. Second, the effect of compatibility must be larger for unexpected than for expected stimuli. Third, subjects must not only expect the stimulus indicated by the hint on each trial, but they must also occasionally expect one or two other stimuli chosen with probabilities proportional to the frequency of occurrences of the various stimuli (cf. Hacker \& Hinrichs, 1974). Together, these assumptions can account for the effect of frequency on unexpected trials and for the interaction of frequency with compatibility on the unexpected trials, entirely in terms of expectancy. In order to demonstrate that frequency also plays a role in producing the probability effect, it is necessary to show either a frequency effect

Table 1

Experiment 1: The Pseudoprobability Effect on Reaction Time (RT, in Milliseconds) and Percentage Correct (PC)

\begin{tabular}{|c|c|c|c|c|c|c|}
\hline \multirow{3}{*}{$\begin{array}{c}\text { S-R } \\
\text { Compatibility }\end{array}$} & \multicolumn{6}{|c|}{ "Probability" } \\
\hline & \multicolumn{2}{|c|}{ High } & \multicolumn{2}{|c|}{ Low } & \multicolumn{2}{|c|}{ Difference } \\
\hline & RT & $\mathrm{PC}$ & RT & PC & $\mathrm{RT}$ & $\mathrm{PC}$ \\
\hline Compatible & 450 & 98.9 & 810 & 91.7 & 360 & 7.2 \\
\hline Incompatible & 478 & 95.1 & 1037 & 85.4 & 559 & 9.7 \\
\hline
\end{tabular}

for expected stimuli or an effect of probability that is not explainable in terms of expectancy.

\section{EXPERIMENT 2}

The purpose of this experiment was to examine the interactions of expectancy, frequency, and visual quality in a paradigm similar to that of Experiment 1. Visual quality is known to interact with stimulus probability (e.g., Miller \& Pachella, 1973). The purpose of this experiment, then, was to separate the interaction of frequency and quality from that of expectancy and quality. A finding that the interaction of probability and quality can be explained in terms of expectancy would further reduce the need for the concept of relative frequency.

A variety of theories and models are based on the assumption that expectancies and other forms of cognitive set can influence perceptual processes (e.g., Bruner, 1957; Rumelhart, 1977; Shiffrin \& Schneider, 1974). According to these theories, the effect of expectancy should be greater for visually degraded stimuli than for normal stimuli. On the other hand, the results of Epstein and Rock (1960) suggest that expectancy does not affect perceptual processing, so its effect should be independent of visual quality. Using the ambiguous wife/mother-in-law figure, Epstein and Rock showed that perception was influenced more by what the observer saw last than by what the observer expected to see next. Since frequent stimuli will, on average, have been seen more recently than infrequent stimuli, this finding supports the hypothesis that visual quality will interact with frequency rather than expectancy.

Two distinct manipulations of visual quality were used in this experiment: contrast reduction and superimposition of random dots. Turvey (1973) has demonstrated that different kinds of visual degradation can have different effects and that the differences can reveal different levels of perceptual processes. In particular, his results suggest that contrast might have an effect at a lower level of visual processing than a superimposed random dot pattern. If so, then the two methods of degradation might interact differentially with expectancy and frequency. Expectancy is clearly a more "top-down" manipulation, requiring active attentional adjustments to produce an effect (cf. Posner, Snyder, \& Davidson, 1980, Experiment 1).

In order to get more information about the influence of the hint procedure on the frequency effect for expected stimuli, this procedure was changed slightly relative to the first experiment. All stimulus letters were used as hints equally often. If no frequency effect is found for expected stimuli using this procedure, it will not be possible to argue that the effect is being hidden by especially complete preparation for letters rarely presented as hints. Such a finding would be strong evidence against the claim that there is a frequency effect 
even for expected stimuli. It should be noted, however, that this change in procedure removes the independence between which hint is presented and whether the hint is correct. Because low-frequency letters were not tested as often as high-frequency letters and because the two types of letters were used as hints equally often, a lowfrequency letter appearing as a hint was less likely than a high-frequency letter to be a correct hint. If the completeness of preparation depends on the probability that a hint is correct, then this dependency could work to produce a frequency effect for expected stimuli in this experiment. Thus, while finding no frequency effect for expected stimuli would be unambiguous evidence against such an effect, finding the effect would not be strong evidence that frequency really does have an effect apart from expectancy.

\section{Method \\ Thirty-six subjects served in this experiment. The details of procedure were nearly identical to those of Experiment 1, with three exceptions. First, the task was character classification (Sternberg, 1969). For each subject, six randomly selected letters were divided evenly between the positive set (right-hand response) and the negative set (left-hand response). Subjects were informed of all six possible letters, but they were told to remember the positive set items and respond with the left hand to any letter not in that set. Second, three different visual conditions were used. In the normal condition, letters were displayed clearly and at high contrast, as in Experiment 1. Contrast reduction was produced by placing a dark plastic screen over the face of the oscilloscope, thus reducing the brightness of the stimulus by about $1.5 \mathrm{log}$ units. Alternatively, stimuli were degraded by superimposing a pattern of random dots over}

the test letters. The dots were single points displayed on the oscilloscope, and their apparent brightness was matched to that of the stimulus letters. A different pattern of random dots was generated for every trial. Third, all stimulus letters were used as hints equally often.

The first block was considered practice and was given under normal visual conditions for all subjects. The remaining three blocks were presented with (1) normal visual conditions, (2) low contrast, and (3) superimposed dots. The order of these three blocks was counterbalanced across subjects.

\section{Results and Discussion}

For each block of trials, the average RT and PC were computed for expected and unexpected triais for all three levels of stimulus frequency. Averages of the RTs across subjects are shown in Figures 4 and 5. An analysis of variance was performed on the RT data, and the main effects of visual quality $[\mathrm{F}(2,70)=59, \mathrm{p}<.01$, MSe $=86,007]$, expectancy $[F(1,35)=125, p<.01$, $\mathrm{MSe}=36,115]$, and stimulus frequency $[\mathrm{F}(2,70)=$ $22.7, \mathrm{p}<.01, \mathrm{MSe}=30,296$ ] were all highly reliable. The only interaction to approach significance was that of frequency and expectancy $[\mathrm{F}(2,70)=3.65, \mathrm{p}<.05$, $\mathrm{MSe}=16,669$ ], with a larger effect of frequency on unexpected than on expected stimuli.

A similar analysis was performed on the PC data, and again the main effects of visual quality $[F(2,70)=$ $10, \mathrm{p}<.01$, MSe $=2,083]$, expectancy $[\mathrm{F}(1,35)=18$, $\mathrm{p}<.01, \mathrm{MSe}=402]$, and frequency $[\mathrm{F}(2,70)=8.7$, $\mathrm{p}<.01, \mathrm{MSe}=231]$ were reliable. In this analysis, however, the interaction of visual quality with stimulus frequency also reached significance $[F(4,140)=3.088$,

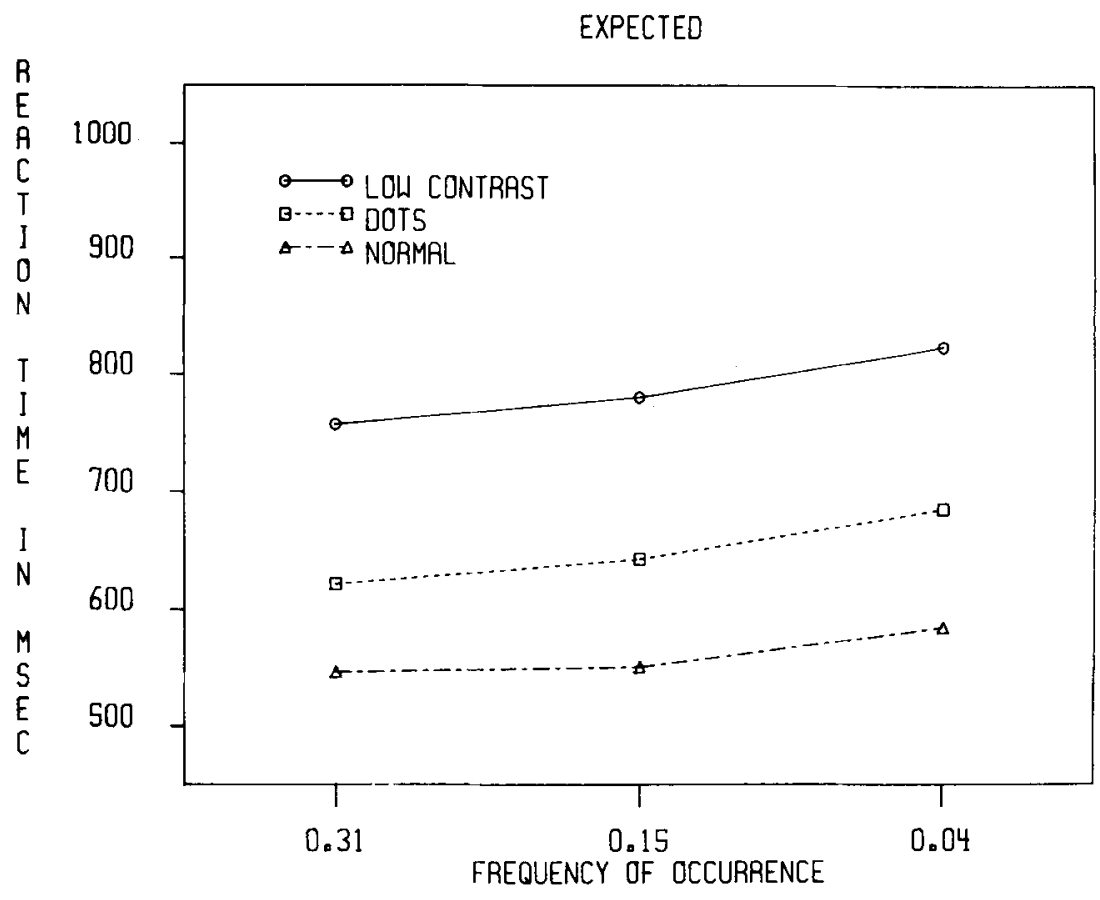

Figure 4. Experiment 2. Expected stimuli. Reaction time as a function of stimulus frequency and visual quality. 


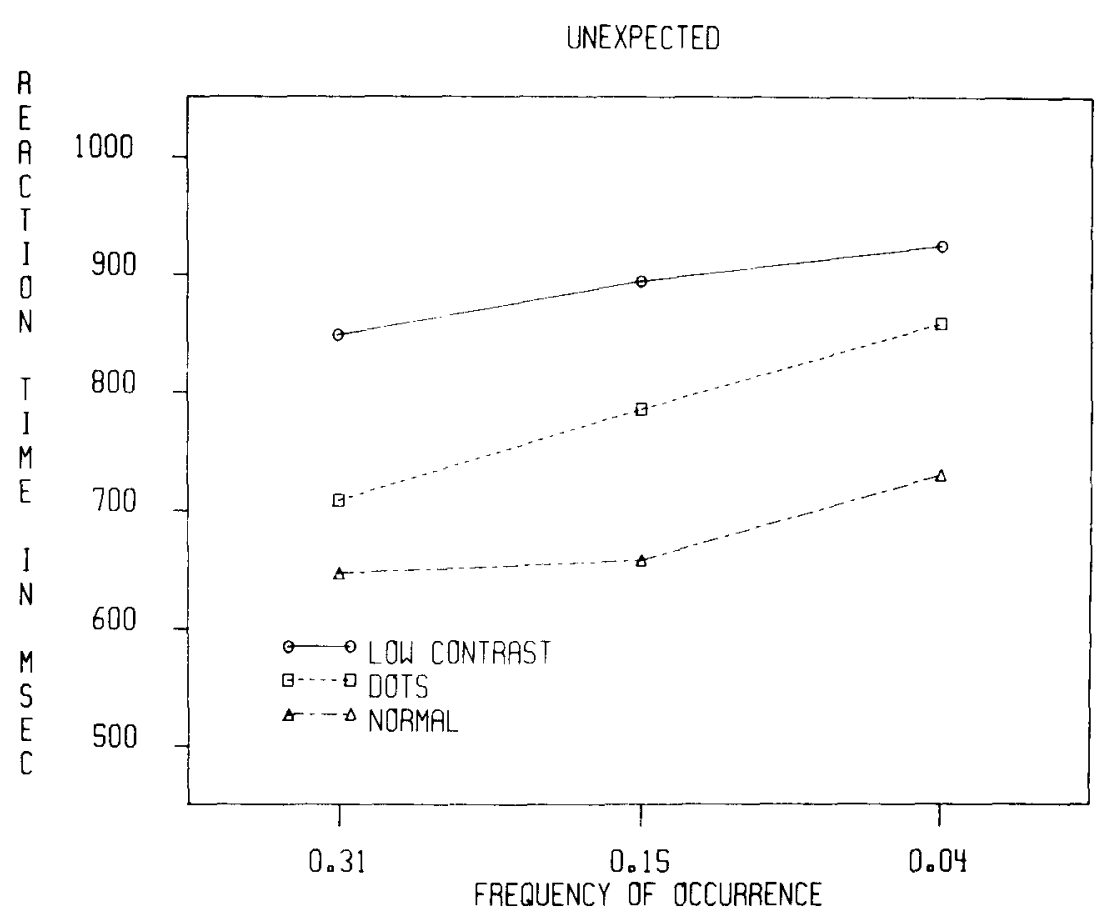

Figure 5. Experiment 2. Unexpected stimuli. Reaction time as a function of stimulus frequency and visual quality.

$p<.025, \mathrm{MSe}=240]$. Average PCs as a function of visual quality and frequency are shown in Table 2 . It is apparent that frequency had a larger influence on error rates in the degraded conditions than in the normal condition. Planned comparisons showed that the effect of frequency was reliably $(\mathrm{p}<.05)$ larger in both degraded conditions than in the normal condition.

The results of this experiment do not support the hypothesis that expectancy influences perceptual processing, since the effect of visual quality did not depend on whether the stimulus was expected or unexpected. Given that this is a null result, it is useful to look at $95 \%$ confidence intervals to see how much change expectancy might actually produce in the effect of visual quality. Overall, the effects of superimposing random dots and reducing contrast were about $97 \mathrm{msec}$ and $218 \mathrm{msec}$, respectively. A confidence interval indicated that the effect of superimposing dots might be as much as $58 \mathrm{msec}$ larger for unexpected stimuli than for expected stimuli, or it might be as much as $26 \mathrm{msec}$ smaller. Similarly, a confidence interval indicated that the effect of contrast reduction might be up

Table 2

Experiment 2: Percentages of Correct Response as a Function of Condition

\begin{tabular}{cccc}
\hline & \multicolumn{3}{c}{ Frequency of Occurrence } \\
\cline { 2 - 4 } $\begin{array}{c}\text { Visual } \\
\text { Condition }\end{array}$ & .31 & .15 & .04 \\
\hline Normal & 97.5 & 95.7 & 97.5 \\
Low Contrast & 95.3 & 94.1 & 90.3 \\
Random Dots & 96.3 & 94.1 & 88.5 \\
\hline
\end{tabular}

to $20 \mathrm{msec}$ larger or $50 \mathrm{msec}$ smaller for unexpected stimuli than for expected stimuli. Thus, while the results do not indicate that expectancy influences effects of visual quality, neither do they completely rule out the possibility of some such influence.

The results do suggest that frequency influences perceptual processing. With respect to error rates, the effect of visual quality was reliably larger for lowfrequency stimuli than for high-frequency stimuli. The pattern of RTs showed the same effect, although the interaction was not statistically reliable. The probability effect was $60 \mathrm{msec}$ in the normal condition, $74 \mathrm{msec}$ in the low-contrast condition, and $107 \mathrm{msec}$ in the dots condition. In view of the well established relation between RT and accuracy (Pachella, 1974), and given that the mean RTs were consistent in direction, these results support the view that changes in stimulus frequency modify the effect of visual quality. Although a dependency between stimulus probability and visual quality has previously been found in RT data (Miller, 1979; Miller \& Pachella, 1973, 1976), it is reasonable to suppose that subjects may have considered themselves to be under more severe time pressure in an experiment with correct hints on $75 \%$ of the trials than in an experiment with no hints at all. As Pachella (1974) has noted, effects that are otherwise found in RT will generally be found in PC when subjects are overtly or covertly put under time pressure. Given the shape of the function relating speed and accuracy (Pachella, 1974), it is reasonable to suppose that if error rates had been about equal in all conditions, the interaction would have been obtained in the RT data. 
Table 3

Experiment 2: The Pseudoprobability Effect on Reaction Time (RT, in Milliseconds) and Percentage Correct (PC)

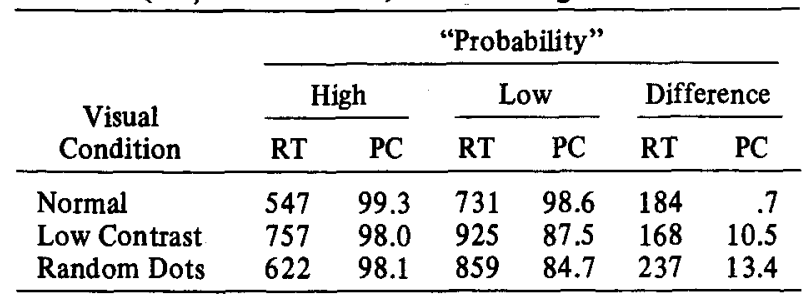

As in Experiment 1, it is possible to try to reconstruct from these data the probability effect observed in tasks in which the subjects are not given hints, comparing high-frequency expected stimuli with low-frequency unexpected stimuli. Table 3 shows the mean RTs and PCs for these stimuli, separately for the three visual conditions. The pattern of results for this pseudoprobability effect is similar to the pattern for the frequency manipulation. Both types of visual degradation increase the pseudoprobability effect with respect to the error rates, but only dots increase the pseudoprobability effect on RT. The additivity of the contrast and pseudoprobability effects on RT could be due to subjects' strategeis and the speed-accuracy tradeoff, as discussed in connection with the nonsignificant interaction of frequency and visual quality on RT.

It is interesting to note that there was an effect of frequency even for expected stimuli in this experiment $[F(2,70)=12.3, p<.01, \mathrm{MSe}=14,206]$. This effect, as noted earlier, does not help to clarify the issue of whether there is an effect of frequency for expected stimuli. However, it can be argued that the issue loses some of its importance in view of the conclusion that expectancy cannot account for the interaction of probability and visual quality. This conclusion implies that frequency is needed to explain some aspects of the probability manipulation and that expectancy cannot be the whole effect.

\section{EXPERIMENT 3}

The purpose of this experiment was to replicate the finding of Experiment 2 that the expectancy effect is not dependent on visual quality. This is a particularly important null result, since it suggests that in this task deliberate attentional adjustments do not affect visual processing. As in the earlier experiments, subjects were given a hint on each trial as to what stimulus would be tested. Stimulus frequency was not varied, however.

\footnotetext{
Method

Twenty-four subjects were run in an experiment that was almost identical to Experiment 2. One major difference was that all letters were tested equally often. The other difference was the addition of a new hint condition. On $40 \%$ of all trials, the hint was the neutral display " + ," which gave no information about what stimulus letter would be presented. On the rest of the trials, the hint was one of the six possible stimulus letters,
}

and $67 \%$ of the hints correctly predicted the test letter. When the test letter was not the same as the hint letter, the test letter was equally likely to require the same response as the hint letter or the other response. Presentation of the hint was followed by a $1.5-\mathrm{sec}$ interval during which the display screen remained blank. Then the test letter was presented, and it remained on the screen until the subject made a response. Following the response, "right" or "wrong" feedback was displayed, and the next hint appeared 2 sec later.

\section{Results and Discussion}

For each block of trials, average RT and PC were computed for each test letter in each of the following four expectancy conditions: (1) The hint had correctly predicted the test letter, (2) the neutral hint had been given, (3) the hint had predicted a letter other than the test letter, but one requiring the same response, and (4) the hint had predicted a letter that required the response other than the one actually required by the test letter.

An analysis of variance on RT was performed, and the main effects of expectancy $[F(3,69)=59, p<.01$, $\mathrm{MSe}=8,385]$ and visual quality $[\mathrm{F}(2,46)=39, \mathrm{p}<.01$, $\mathrm{MSe}=30,587]$ were significant. The expectancy effect was entirely accounted for by an advantage in the correct hint condition $(587 \mathrm{msec})$, and responses in the other three conditions did not differ $(704,708$, and $699 \mathrm{msec}$, respectively). The interaction of expectancy and quality did not approach significance $[F(6,138)=$ $.75, \mathrm{p}>.10, \mathrm{MSe}=5,637]$. Figure 6 shows the average RT as a function of visual quality and expectancy, averaging together the expectancy conditions with neutral and incorrect hints. Confidence intervals were computed to see how the effects of visual conditions depended on whether a stimulus was expected or unexpected, with results similar to those of Experiment 2. In an analysis of variance on PCs, only the factor of expectancy was significant $[\mathrm{F}(3,69)=6.8, \mathrm{p}<.01, \mathrm{MSe}=$ 69.6]. The PCs corresponding to the four expectancy conditions were (1) $98.2 \%$, (2) $96.1 \%$, (3) $93.9 \%$, and (4) $95.3 \%$, with a difference of $2.6 \%$ being significant by a Newman-Keuls test.

It is apparent that there is a great advantage resulting from presentation of a correct hint, as compared with no hint or an incorrect hint. There is no evidence, however, that any of this advantage is due to preparations in visual processing. Degrading the stimulus produced the same effect whether the stimulus was expected or not, so it appears that the hint was used to speed processes subsequent to stimulus identification rather than to speed the recognition process itself. This finding suggests that expectancy plays little or no role in perceptual adjustments made to stimuli of varying probability.

It is interesting to note that the neutral hint was no better than an incorrect hint. One explanation for this finding is that subjects randomly generated an expectation of their own on neutral hint trials. These expectations would have been incorrect $5 / 6$ of the time, and the 


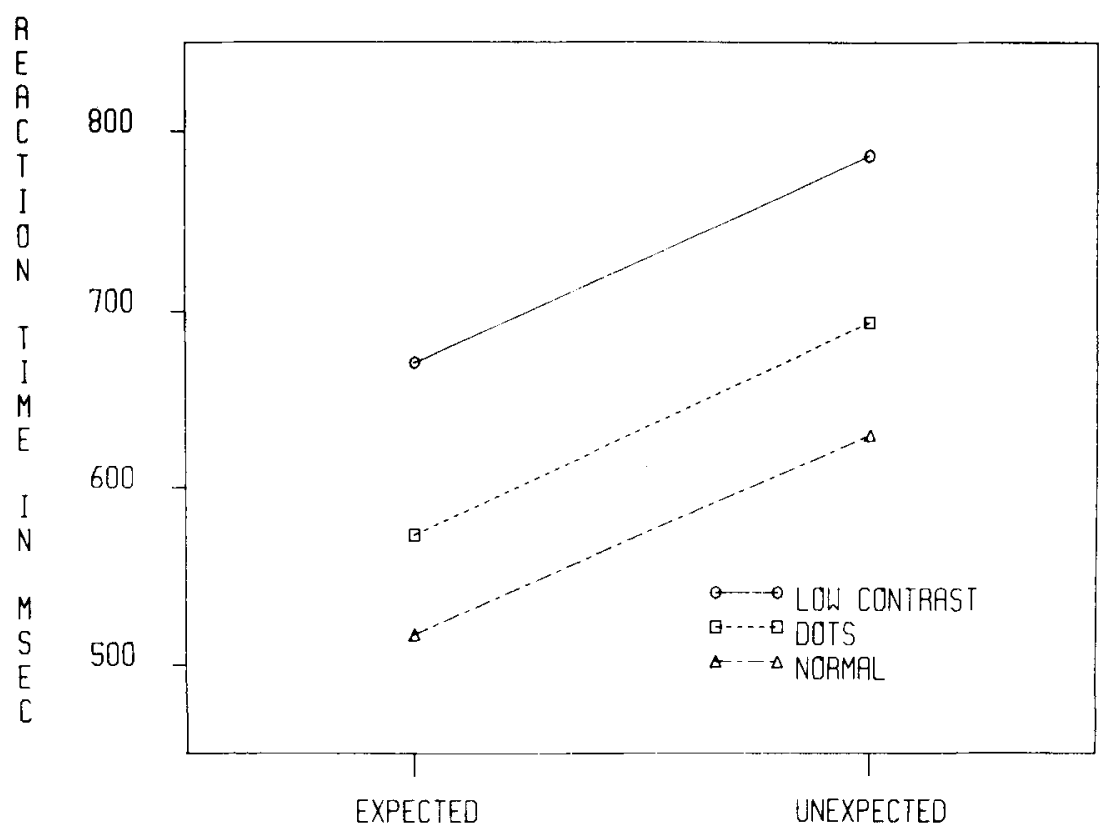

Figure 6. Experiment 3. Reaction time as a function of expectancy and visual quality.

experiment may not have been sufficiently powerful to detect the difference between this condition and the conditions in which the expectations were wrong $100 \%$ of the time. In support of this explanation, it should be noted that LaBerge et al. (1970) found that incorrect hints produced inhibition. It is also possible, however, that correct hints may facilitate the decision making process, even when incorrect hints do not inhibit or mislead it. Becker (Note 1) obtained a similar result in a lexical decision task. He found that in blocks of trials in which the majority of semantic associates were strong synonym or antonym relationships, there was a large benefit but no cost for priming with semantic context. He suggested that subjects used a strategy of explicit anticipation of semantic associates of the context item in these blocks, a strategy closely related to the anticipation we are suggesting occurs as a result of the hint.

\section{GENERAL DISCUSSION}

The results of these experiments indicate that there are important differences between expectancy effects and frequency effects and that both factors are important in explaining the effect of stimulus probability. Both expectancy and frequency influence overall RT, but they have different interactions with other experimental factors. The effect of S-R compatibility is much larger for unexpected than for expected stimuli, but the effect of visual quality is independent of whether a stimulus is expected. Stimulus frequency also influences the effect of S-R compatibility, although less so than expectancy. Also, the effect of visual quality is larger for low-frequency stimuli than for high-frequency stimuli.

The large interaction of expectancy with compatibility suggests that expectancy affects performance primarily by allowing preparation of response selection processes. Whatever the problem is in activating an incompatible response, it is clear that the opportunity to prepare is a great help. This supports the view that the compatibility effect rests on the problem of using a stimulus code to activate a response code. If preparation is allowed, the response code can be activated in advance, thus speeding the response when the correct stimulus is presented. Response preparation is quite specific, however, in the sense that a response is prepared for a given stimulus. There is no advantage in preparing a response to one stimulus and then making that response to a different stimulus (Experiment 3). Thus, with respect to the question of whether we have expectations to perceive a particular stimulus or to execute a particular response (e.g., Hinrichs \& Krainz, 1970), the answer from these results seems to be that neither view is correct. Rather, we expect to execute a particular response to a particular stimulus.

Contrary to models in which the perceptual system somehow gives special activation to an expected item, the present studies have not specifically indicated any perceptual effect of expectancy. It appears that the time needed to recognize a letter presented under viewing conditions adequate for near-perfect recognition does not depend on whether the letter is expected, although, of course, the time needed to respond to the letter does depend on expectancy. These results suggest that the interaction of probability with visual quality is not a 
by-product of the effect of probability on expectancy but, rather, arises from the frequency aspect of the probability manipulation. The results are also relevant to the long debate about the effects of set on perception carried out using tachistoscopic presentation (e.g., Pachella, 1975). The present results indicate that expectancy does not influence the rate at which discriminative information is extracted from the stimulus, thus supporting the view that expectancy has biasing effects rather than facilitating information extraction (cf. Lawrence \& Coles, 1954). The conclusion that expectancy does not facilitate perceptual processes should not be generalized too far, however, since it is easy to think of other tasks in which such an effect would be found. Consider, for example, a task in which stimuli were presented in different locations. Expectancies with respect to the location in which a stimulus would be presented have a perceptual effect in such a task, simply by causing the subject to focus attention on the expected location (Shulman, Remington, \& McLean, 1979).

Several studies have found no effect of stimulus probability with highly compatible S-R mappings (e.g., Hawkins et al., 1973; Posner, 1966; Theios, Note 2). If stimulus frequency affects perceptual processing, as argued here, then stimulus probability effects would be expected even with high S-R compatibility. Although probability effects might be reduced under such conditions, they should not be eliminated entirely. One plausible resolution of this apparent inconsistency is that there is always a probability effect, but that it is small and hard to detect when compatibility is high. Several facts support this analysis. First, studies failing to find a probability effect have not included lowprobability items $(p<.05)$. This is an important consideration, because the effect of probability is highly nonlinear and only items at very low probabilities are greatly delayed. Second, responses to low-probability items were generally slower than responses to highprobability items, even though the effects were not statistically reliable. Finally, there has occasionally been evidence of probability effects in the error rates even though there was no such evidence in the RTs (cf. Pachella's, 1974, discussion of Theios, Note 2). These considerations, together with the usual cautions against accepting a null hypothesis, suggest that there is really no good reason to believe that the probability effect ever disappears completely.

The identification of expectancy as a specific component of the probability manipulation helps to account more explicitly for several findings concerning the effect of probability. For example, preparation could well be the factor mediating the finding of Broadbent and Gregory (1965) that compatibility interacts with response bias. Frequent responses would be expected and prepared for, reducing the compatibility effect, as in Experiment 1. As another example, two studies of the memory scanning task have shown that subjects' strategies for using positive and negative sets may depend on perceived stimulus probabilities and that the effects of probability may depend on these strategies (Briggs, Thomason, \& Hagman, 1978; Hawkins \& Hosking, 1969). It seems clear that the important manipulation in these studies is the manipulation of subjects' expectancies. Subjects may come to expect certain items and base their use of positive and negative sets on these expectations, thereby modifying effects of probability in the two sets.

In the light of the effects of expectancy and frequency on RT, it is tempting to try to account for the effect of stimulus probability as the sum of two effects. First, suppose that the function of expectancy is to call conscious attention to specific high-probability stimuli. This attention could activate specific S-R pathways for these stimuli, but it does not seem to influence perceptual processing. A second factor, practice, gradually and unconsciously strengthens S-R pathways for high-frequency stimuli and also facilitates perceptual mechanisms responsible for the recognition of these stimuli. Such a two-component approach to the nature of probability effects seems promising for furthering our understanding of the complex phenomena.

\section{REFERENCE NOTES}

1. Becker, C. The costs and benefits of semantic context in visual word recognition. Paper presented at the 19 th annual meeting of the Psychonomic Society, San Antonio, Tex., November 1978.

2. Theios, J. The locus of cognition. Paper presented at the 13th annual meeting of the Psychonomic Society, St. Louis, November 1972.

\section{REFERENCES}

Beller, H. Priming: Effects of advance information on matching. Journal of Experimental Psychology, 1971, 87, 176-182.

Bernstein, I., \& Reese, C. Behavioral hypothesis and choice reaction time. Psychonomic Science, 1965, 3, 259-260.

Bernstein, I., Schurman, D., \& Forester, G. Choice reaction time as a function of stimulus uncertainty, response uncertainty, and behavioral hypotheses. Journal of Experimental Psychology, $1967,74,517-524$.

Bertelson, P., \& Tisseyre, R. Choice reaction time as a function of stimulus versus response relative frequency of occurrence. Nature, 1966, 212, 1069-1070.

Biederman, I., \& Zachary, R. Stimulus versus response probability in choice reaction time. Perception \& Psychophysics, $1970,7,189-192$.

Buackman, A. Test of the additive-factor method of choice reaction time analysis. Perceptual Motor Skills, 1975, 41, $607-613$.

Brioos, G., Thomason, S., \& Hagman, J. Stimulus classification strategies in an information reduction task. Journal of Experimental Psychology: General, 1978, 107, 159-186.

Brondene, D., \& Gregory, M. On the interaction of S-R compatibility with other variables affecting reaction time. British Journal of Psychology, 1965, 56, 61-67. 
Bruner, J. On perceptual readiness. Psychological Review, 1957, 64, 123-152.

Dillon, P. Stimulus versus response decisions as determinants of the relative frequency effect in disjunctive reaction time performance. Journal of Experimental Psychology, 1966, 71, 321-330.

Epste in, W., \& Rock, I. Perceptual set as an artifact of recency. American Journal of Psychology, 1960, 73, 214-228.

Estes, W. Probability learning. In A. W. Melton (Ed.), Categories of human learning. New York: Academic Press, 1964.

FirTs, P. Perceptual-motor skill learning. In A. Melton (Ed.), Categories of human learning. New York: Academic Press, 1964.

Fitss, P., Peterson, J., \& Wolfe, G. Cognitive aspects of information processing: II. Adjustments to stimulus redundancy. Journal of Experimental Psychology, 1963, 65, 423-432.

Geller, E., \& Pitz, G. Effects of prediction, probability, and run lengths on choice reaction speed. Journal of Experimental Psychology, 1970, 84, 361-367.

Geller, E., Whitman, C., \& Post, D. Stimulus probability and prediction outcome as determinant of choice reaction time: Some procedural considerations. Acta Psychologica, 1973, 37, 1-14.

Geller, E., Whitman, C., Wrenn, R., \& Shipley, W. Expectancy and discrete reaction time in a probability reversal design. Journal of Experimental Psychology, 1971, 90, 113-119.

Hacken, M., \& Hinrichs, J. Multiple predictions in choice reaction time: A serial memory scanning interpretation. Journal of Experimental Psychology, 1974, 103, 999-1005.

Hawkins, H., \& Hosking, K. Stimulus probability as a determinant of discrete choice reaction time. Journal of Experimental Psychology, 1969, 82, 435-440.

Hawkins, H., Mackay, S., Holley, S., Friedin, B., \& CoHEN, S. Locus of the relative frequency effect in choice reaction time. Journal of Experimental Psychology, 1973, 101, 90-99.

Hawkins, H., Thomas, G., \& Dhury, K. Perceptual versus motor bias in discrete reaction time. Journal of Experimental Psychology, 1970, 84, 514-517.

Hinrichs, J. Probability and expectancy in two-choice reaction time. Psychonomic Science, 1970, 21, 227-228.

HinRichs, J., \& Craft, J. Verbal expectancy and probability in two-choice reaction time. Journal of Experimental Psychology, $1971,88,367-371$.

Hinaichs, J., \& Krainz, P. Expectancy in choice reaction time: Anticipation of stimulus or response? Journal of Experimental Psychology, 1970, 85, 330-334.

Hyman, R. Stimulus information as a determinant of reaction time. Journal of Experimental Psychology, 1953, 45, 188-196.

KEELE, S. Repetition effect: A memory-dependent process. Journal of Experimental Psychology, 1969, 80, 243-248.

KLATZKY, R., \& Smith, E. Stimulus expectancy and retrieval from short-term memory. Journal of Experimental Psychology, 1972, 94, 101-107.

LaBerge, D., Legrand, R., \& Hobbie, R. Functional identification of perceptual and response biases in choice reaction time. Journal of Experimental Psychology, 1969, 79, $295-299$.

LABenge, D., \& Tweedy, J. Presentation probability and choice time. Journal of Experimental Psychology, 1964, 68, 477-481.

LABerge, D., Van Gelder, P., \& Yelotot, J. A cueing technique in choice reaction time. Perception \& Psychophysics, 1970, 7, 57-62.
LAMING, D. Subjective probability in choice-reaction experiments. Journal of Mathematical Probability, 1969, 6, 81-120.

Lawrence, D., \& Coles, G. Accuracy of recognition with alternatives before and after the stimulus. Journal of Experimental Psychology, 1954, 47, 208-214.

MiLLER, J. Cognitive influences on perceptual processes. Journal of Experimental Psychology: Human Perception and Performance, 1979, 5, 546-562.

Miller, J., \& Pachella, R. On the locus of the stimulus probability effect. Journal of Experimental Psychology, 1973, 101, 227-231.

Miller, J., \& Pachella, R. Encoding processes in memory scanning tasks. Memory \& Cognition, 1976, 4, 501-506.

NeELY, J. Semantic priming and retrieval from lexical memory: Roles of inhibitionless spreading activation and limited-capacity attention. Journal of Experimental Psychology: General, 1977, 106, 226-254.

Orenstein, H. Reaction time as a function of perceptual bias, response bias, and stimulus discriminability. Journal of Experimental Psychology, 1970, 86, 38-42.

Pachella, $R$. The interpretation of reaction time in information processing research. In B. Kantowitz (Ed.), Human information processing: Tutorials in performance and cognition. Hillsdale, N.J: Erlbaum, 1974

Pachella, R. The effect of set on the tachistoscopic recognition of pictures. In P. Rabbitt \& S. Dornic (Eds.), Attention and performance $V$. New York: Academic Press, 1975.

Posner, M. Components of skilled performance. Science, 1966, 152, 1712-1718.

Posner, M., Snyder, C., \& Davidson, B. Attention and the detection of signals. Journal of Experimental Psychology: General, 1980, 109, 160-174.

Rosenbaum, D. Human movement initiation: Specification of arm, direction, and extent. Journal of Experimental Psychology: General, 1980, 109, 444-474.

Rumelhart, D. Toward an interactive model of reading. In S. Dornic \& P. Rabbitt (Eds.), Attention and performance VI. New York: Academic Press, 1977.

SANDERS, A. Some variables affecting the relation between relative stimulus frequency and choice reaction time. Acta Psychologica, $1970,33,45-55$.

Shiffrin, R., \& Schneider, W. An expectancy model for memory search. Memory \& Cognition, 1974, 2, 616-628.

Shulman, G., Remington, R., \& Mclean, J. Moving attention through visual space. Journal of Experimental Psychology: Human Perception and Performance, 1979, 5, 522-526.

SPECTOR, A., \& LyONs, R. The locus of the stimulus probability effect in choice reaction time. Bulletin of the Psychonomic Society, 1976, 7, 519-521.

Stennberg, S. Memory scanning: Mental processes revealed by reaction-time experiments. American Scientist, 1969, 57, 421-457.

Theio8, J., Smith, P., Haviland, S., Traupmann, J., \& MoY, M. Memory scanning as a serial self-terminating process. Journal of Experimental Psychology, 1973, 97, 323-336.

TURvey, M. On peripheral and central processes in vision: Inferences from an information-processing analysis of masking with patterned stimuli. Psychological Review, 1973, 80, 1-52.

(Received for publication December 8, 1980; revision accepted May 5, 1981.) 